Elisabeth Knipf-Komlósi

ELTE, Budapest

\title{
Zur Frage des Normverständnisses im DaF-Unterricht
}

\author{
DOI: 10.14232/fest.bassola.28
}

\begin{abstract}
Es gilt als Binsenwahrheit, dass eine gut ausgebildete Sprachkompetenz/Sprachhandlungskompetenz der Schlüssel zu einer erfolgreichen Partizipation am gesellschaftlichen und sozialen Leben ist. Die Schule trägt neben anderen Instanzen selbstverständlich auch in großem Maße die Verantwortung dafür, dass diese Sprach(handlungs)kompetenz auf bestmöglichem Wege, mit den angemessenen Methoden bei den Deutschlernenden ausgebaut wird. Die deutsche Sprache ist keine leicht zu erlernende Sprache, und hier wird häufig als eines von vielen Argumenten angeführt, dass Deutsch eine der variationsreichsten Sprachen Europas ist, die zudem in die Reihe der plurizentrischen Sprachen, so wie die großen Sprachen Europas, Englisch, Französisch, Spanisch etc. gehört. Im vorliegenden Beitrag wird der Frage nachgegangen, welche Überlegungen - theoretische, wie die Frage der Plurizentrik, die zentrale Frage der Norm(en), wie die curricale Gestaltung des DaF- Studiums - die Grundlage bei der Entwicklung eines Normverständnisses bei Lehrenden wie Lernenden eine Rolle spielen können/sollen, um eine positive, wenig frustrierende Einstellung zum Deutschlernen zu erreichen ${ }^{1}$. In diesem Sinne gliedert sich mein Beitrag in die Fragen der Norm-Debatte, in die Gründe der Norm-Debatte in der Auslandsgermanistik bzw. in die kurze Darstellung einer empirischen Abfrage zum Thema mit einem Ausblick.
\end{abstract}

\section{Fragen um die Norm-Debatte}

Von einem theoretischen Standpunkt aus soll mit einer allgemeineren Norm-Definition begonnen werden, welche wichtige Aspekte der Norm beleuchtet:

Die Frage der Norm-Debatte wurde in letzter Zeit in mehreren empirischen Forschungen im DaF-Bereich in Europa aufgegriffen vgl. Davies (2006) und Davies / Langer (2013), Durrell (2006), Neuland (2006), Stridova (2015) u.v.m. 
Eine Sprachnorm ist ein historisch veränderlicher und aufgrund der Reflexion sozialer Phänomene intersubjektiv existierender Bewusstseinsinhalt, der als Regulator sprachlicher Erwartungen und Handlungen funktioniert und der sich auf gleichartige und zahlenmäßig nicht näher bestimmbare Kommunikationssituationen bezieht. Die Verletzung der Sprachnorm beschert dem Sprachbenutzer gewisse (negative) Folgen (Dovalil 2006: 26).

Der einzig wunde Punkt dieser Definition liegt m.E. in der Bestimmung der Begrenzung der regulativen Funktion der Sprachnormen auf „gleichartige und zahlenmäßig nicht näher bestimmbare Kommunikationssituationen“, die in ihrer vagen Formulierung keine eindeutigen Anhaltspunkte für die gemeinten Kommunikationssituationen geben können. In der einschlägigen Fachliteratur gibt es vielfach Diskussionen über die Normiertheit von medial unterschiedlich realisierten Sprachhandlungen, über den Normierungsprozess selbst bzw. auch über die in letzter Zeit immer öfter auftauchende Diskussion über die Flexibilisierung von Normen. Die letzteren zwei Fragen hängen aufs engste mit empirischen Untersuchungen zu Sprachgebrauchssituationen zusammen und haben Auswirkungen z.B. auch auf den Umgang mit sprachlichen Normen in der Schule auf dem Sprachgebiet und auch im DaF-Bereich im Ausland.

Seit der pragmatischen Wende der 60er und 70er Jahre in Deutschland wird ein flexibler Zugang an sprachliche Normen, das Erkennen ihrer Veränderlichkeit angestrebt, denn bis dahin hielt man an den eindeutig kodifizierten normativen Grammatiken fest, an deren Beständigkeit man - ohne Rücksicht auf die Veränderungen der Sprachrealität - fest glaubte und sich konsequent daran hielt. Im Allgemeinbewusstsein sind nämlich Normen primär inhaltlich festgelegte Regulative für die einzelnen Sprachebenen, die orthographische, orthoepische, grammatische (morphologische, syntaktische), und die lexikalisch-semantische Ebene. Gloy (1998) bezeichnet diese als konventionelle, gesetzte Normen, die als Ordnungsprinzip beim Sprachgebrauch des Individuums oder der Sprachgemeinschaft mehr oder weniger verbindlich sind. Doch wie sieht die Verbindlichkeit dieser Normen bei einer so heterogenen und plurizentrischen Sprache wie dem Deutschen aus, mit so vielen Subsystemen und arealen, sozialen und funktionalen Varianten, insbesondere in der gesprochenen Sprache, in der durch die starke Betonung der Varianz in der Gegenwart 
der Normativitätsaspekt in den Hintergrund geraten ist? Bereits vor zwei Jahrzehnten entstand der Bedarf nach einer anderen, viel differenzierteren Sicht auf die Norm und auf deren Legitimierung:

Von besonderer Bedeutung erscheinen neben den kodifizierten und statuierten Normen nun die Entwicklungen der, subsistenten' Normen, jener normativen Erwartungshaltung, um deren Institutionalisierung und Legitimierung im Rahmen konkurrierender Normierungshandlungen gerungen wird (Neuland 1998: 8).

Daher ist zu begrüßen, dass in der einschlägigen Fachdiskussion der letzten Jahre bereits das Begriffspaar ,statuierte' (explizite) und ,subsistente' (verdeckte, implizite) Normen im Mittelpunkt der Diskussion steht (vgl. Gloy 2004, Ziegler 2011). Diese zwei Typen von Normen sind hinsichtlich des Sprachgebrauchs zwar eng miteinander verflochten, doch in ihrer Entstehung und Funktion sehr unterschiedlich, und sie werden auch auf unterschiedliche Weise gehandhabt. Die statuierten Normen gelten als kodifizierte und tradierte Normen, die als sichere Orientierung für Lehrende und Lernende dienen, verbindlich im Schulunterricht sind und ein gesellschaftlich hohes Prestige genießen. Die verdeckten, sog. subsistenten Normen erscheinen in konkreten Sprachgebrauchssituationen, widerspiegeln das kommunikative Verhalten der sprechenden Personen und beziehen sich auf sprachliche Handlungsroutinen. Da sie nicht geregelt und kodifiziert sind, besitzen sie kein hohes Prestige, zumal sie noch als Abweichungen von der Standardnorm betrachtet werden (vgl. Ziegler 2011: 70ff.).

Theoretisch umfassen die beiden Normen den Gegenstand eines komplexen Normbegriffs sowie dessen Anwendung, den Sprachgebrauch. Die Sprachnormen sind bestimmend für alle Bereiche der Linguistik, die sich mit dem Sprachgebrauch beschäftigen. Feilke (2015: 118) z.B. erweitert den Horizont der Normen um eine didaktische Dimension und hebt drei Ebenen des Problems hervor, nämlich die Normativität in der Didaktik, die Normativität des Fachs, d.h. die sprachlichen Normen, und zuletzt nimmt er noch Normen für den Unterricht an. Bei letzteren geht es um „Normen als kommunikatives und kognitives Regulativ für Lehr- und Lernprozesse“. Es bedarf keiner besonderen Einsicht, dass in einem modernen Deutschunterricht über die gesetzten Normen hinaus - die als der Gegenstand des Unterrichts sowieso im Vordergrund stehen - auch die 
Gebrauchsnormen sowie jenes kommunikative und kognitive Regulativ ${ }^{2}$ im Unterricht eine wichtige Rolle einnehmen müssen, denn das primäre Ziel der Lehrenden wie Lernenden ist es, sich einen in vielen Situationen handhabbaren Sprachgebrauch anzueignen. So kann man mit Durrell (2012: 103), der aus der Sicht der Auslandsgermanistik das Problem betrachtet, einverstanden sein, dass

die ausschließliche Vermittlung der herkömmlichen hochsprachlichen Normen heutzutage nicht mehr für die Vermittlung umfassender fremdsprachlicher Kompetenzen ausreicht, denn die Lernenden müssen sich auch die Fähigkeit aneignen, sich an einer Vielfalt sprachlicher Handlungen in angemessener Weise zu beteiligen.

Die drei Grundpfeiler des Begriffs lassen auf die Komplexität des Begriffs schließen: Zum einen geht es um eine Komponente der Umstände und Bedingungen, die zu einer Norm führen (Antezedens), zweitens geht es um die Auffassung der zu realisierenden normgerechten Sprachhandlung (Implikat) sowie drittens um Sanktionen, die dann eintreten, wenn die durch die Norm implizierte Handlung nicht eintritt, obwohl die Bedingungen dazu gegeben sind (vgl. Dovalil 2010: 47). Bei den drei Komponenten geht es nicht um eine starre, einzig mögliche sprachliche Handlungsform, vielmehr wird hier mit der Variation gerechnet und dadurch der Norm eine elastische Stabilität zugeschrieben.

Darüber hinaus haben wir es beim Begriff der Sprachnorm mit einem relationalen Begriff zu tun, der erst durch eine Inbezugsetzung mit komplementären Begriffen sein volles Bedeutungsspektrum zum Ausdruck bringen kann. So muss über eine Norm im Zusammenhang mit dem Sprachgebrauch eines Individuums oder einer Gemeinschaft gesprochen werden, doch vor allem muss das Phänomen der sprachlichen Variation, der angemessenen Wahl von möglichen Optionen in der Sprachhandlung vor dem Hintergrund eines Norm-Begriffs diskutiert werden. Das bestehende Spannungsverhältnis zwischen Normbefolgung und Normabweichung, auch der Normtoleranz, und dergleichen wichtige Zusammenhänge können erst durch ihre Beziehung zum Normbegriff erläutert und ins rechte Licht gerückt werden.

\footnotetext{
2 Die von Feilke genannte dritte Norm verstehe ich als Teil der versteckten (subsistenten) Normen auf der Metaebene.
} 


\section{Curriculare Fragen des Deutschen in der Auslandsgermanistik und deren Anwendung $^{3}$}

Aus der Sicht der Auslandsgermanistik und im DaF-Bereich schlechthin kann von folgender Situation ausgegangen werden. Aufgrund der gängigen und z.Z. aktuell gültigen curricularen Vorgaben der Auslandsgermanistik in Ungarn erlernen angehende Lehrerinnen und Lehrer des Deutschen im Laufe ihrer Ausbildung die Sprache als Kommunikationsmittel (Sprachgebrauch) auf einem entsprechend hohen Niveau des Gemeinsamen Europäischen Referenzrahmens parallel mit einer linguistischen Kompetenz, und den gesetzten Normen, besonders in den Bereichen der Orthographie und Grammatik. Die Regeln des Sprachsystems der Standardsprache, die expliziten Normen, sind in Wörterbüchern, Lehr- und Sprachbüchern sowie Grammatiken kodifiziert, übersichtlich dargestellt und didaktisch gut aufbereitet. Im Lehramtsstudium für Deutsch bilden diese kodifizierten Normen die wichtigste Stütze im Schulunterricht, die gut didaktisiert und in der Lehre mehr oder weniger leicht vermittelbar sind ${ }^{4}$. Aufgrund der bereits angeeigneten sprachlichen Handlungsmuster müssen auch die Sprachgebrauchsnormen kennen gelernt werden, vor allem jene, die durch diverse Kommunikationssituationen in der Lehre und in den Lehrbüchern vermittelt werden. Im Vergleich zu den statuierten Normen sind die verdeckten, impliziten Normen nicht als Regelwerk vorgegeben und erlernbar, sie sind auch in schulischen Kommunikationssituationen nicht so einfach erfahrbar, denn oft fehlt es an konkreten Sprechanlässen und Kommunikationssituationen, die - wie das aus den Erfahrungen der Lehrenden hervorgeht - im Fremdsprachenunterricht mangels entsprechender kulturell-sozial bedingter Kenntnisse nicht so leicht gemeistert werden können. Es geht eben um jene Routinen, denen im heutigen Fremdsprachenunterricht genauso viel Relevanz zukommen sollte wie den Regularitäten des Sprachsystems. Bedenken werden diesbezüglich auch im Vorwort des Tagungsbandes zur IDS-Jahrestagung 2009

\footnotetext{
An zweisprachigen Gymnasien mit muttersprachlichen Lehrenden sind die Sprachhandlungsroutinen der Lernenden selbstverständlich ausgeprägter und differenzierter als in einem einfachen Fremdsprachenunterricht (vgl. Müller 2010: 74-96 und Berend / Knipf 2006: 161-175). $4 \mathrm{Zu}$ den grammatischen Regelwerken, die von Germanistikstudierenden gebraucht werden vgl. Miskei / Müller 2017: 119-133).
} 
formuliert, nämlich, dass es noch unklar ist „wie ein Konzept zum Sprachgebrauch und seiner Ermittlung auszusehen habe, um mit der Vorstellung von Regularitäten kompatibel zu sein“ (Eichinger 2009: 2).

Seit sprachwissenschaftliche Forschungen als Gegenstand ihrer Untersuchungen auch die gesprochene Sprache bzw. gesprochene Varietäten des Deutschen in ihr Fokus stellen, ergab sich in der Auslandsgermanistik eine zusätzliche Aufgabe, deren Vermittlung mitunter zu Schwierigkeiten führen kann. Durch die im gesprochenen Deutsch auftauchenden variablen Abweichungen von den bislang gelernten statuierten Normen tut sich eine Lücke auf, eine wahrnehmbare Ungewissheit in der Relation Sprachgebrauch und Norm im DaF-Bereich, wenn nämlich Lernende, Studierende sprechsprachliche Formen im Alltagsdeutsch wie z.B. is statt ist, sonen statt so einen, inner Klasse statt in einer Klasse und dergleichen mehr zu hören bekommen, da gewisse Normen in einer aufgelockerten Form im sprachlichen Umgang des Alltagslebens nicht immer konsequent durchgesetzt werden. Das Prinzip der sprachlichen Korrektheit und Angemessenheit sowie das der systematischen Konsequenz sind zwar Grundelemente einer sprachnormativen Haltung, die jedoch „keineswegs eine kulturhistorische Selbstverständlichkeit“ (Busse 2006: 3-4) sind. Diese Haltung beruht auf den Einstellungen der Mitglieder der Gesellschaft zur ihrer Sprache, die dann als Haltung auch für DaF-Lerner beispielgebend wirkt. Ziel des Unterrichts sollte im Ausland sein, den Lernenden bestimmte, im sprachlichen Alltag wirkende Gebrauchsnormen von Muttersprachlern auch zu erschließen und zu vermitteln. Die Erschließung der Gebrauchsnormen führt über die Erfahrung mit der Kultur und Sprache des Deutschen. Angesichts dieser Zielstellung sollte eine grundlegend andere Sicht in der Deutschlehrerausbildung auf curricularer Ebene ansetzen, indem versucht wird, die relevanten sozialen Normen der Kultur der betreffenden Sprache im Unterricht so einzubauen und zu vermitteln, dass diese die Aufmerksamkeit der Lernenden wecken und zum besseren Verständnis und einer allmählichen Adaption des sich verändernden Sprachgebrauchs beitragen können.

Das Hochschulcurriculum in Germanistik bietet die Möglichkeit, dass sich die Studierenden in fachlichen Diskussionen zum Thema der sprachlichen Norm der Gegenwart im Laufe ihres Studiums oder bei Fortbildungen ausei- 
nandergesetzt haben ${ }^{5}$, genauso wie mit dem Konzept der Plurizentrik und der Variationsvielfalt des Deutschen. Seit den 90er Jahren wird - auch aufgrund der curricularen Vorgabe - die Plurizentrik und die sprachliche Variation des Deutschen aus soziolinguistischer Sicht immer mehr thematisiert, in Fachaufsätzen und Diskussionen bearbeitet. Doch sind diese zwei Schwerpunkte mit all ihren Facetten noch keine selbst erfahrene und erlebte Sprachwirklichkeit für die meisten Studierenden und Lernenden im Ausland.

Bedauerlich ist auch, dass im Curriculum der Deutschlehrerausbildung (in Ungarn) die Frage der Norm bislang keine besondere Gewichtung bekam. Dies kann mehrere Gründe haben:

(1) Für Lehrende, Lernende und Studierende ist es nicht selbstverständlich, dass sie Informationen über die Veränderungen der Gebrauchsnormen der Gegenwart erfahren können; die wenigen Lehrenden und Lernenden, die mehr motiviert und sprachgewandt sind, haben diese Kenntnisse oft nur mühsam selbst zusammengetragen. Der variable Sprachgebrauch als Begriff ist ihnen zwar vertraut, doch dass jede sprachliche Äußerung durch die Situation, Funktion, Gesprächspartner etc. motiviert ist, und dadurch eine große sprachliche Variabilität entsteht, bleibt doch eher ein theoretisches Konstrukt.

(2) Die Sensibilität der Deutschlehrenden und -lernenden im Ausland für die breitgefächerte Vielfalt sprachlicher Handlungs- und Textmuster der Gegenwart in der Vielfalt der Medientextsorten und im sozialen Umfeld des öffentlichen Lebens ist verständlicherweise bei weitem nicht so ausgeprägt und kann nicht so bewusst gebraucht werden wie bei Muttersprachlern oder den Deutsch Lehrenden im Sprachgebiet. Demzufolge ist das Variations- und Normempfinden der DaF-Lehrenden/Studierenden selbstverständlich auch different.

\footnotetext{
$5 \quad$ Z.B. in Form von philologischen Modulen, wie Sprachsystem und Sprachwandel, Variation und Varietäten des Deutschen, Soziolinguistik des Deutschen und dergleichen mehr thematisieren (die Beispiele stammen aus dem Curriculum des Germanistischen Instituts der Eötvös-Lorand-Universität Budapest).
} 
(3) Aus o.g. Gründen steht daher im Zentrum des DaF-Unterrichts überwiegend noch die Vermittlung der kodifizierten Normen ${ }^{6}$, auch wenn eine gewisse Lockerung in dieser Hinsicht z.B. im Rahmen der Diskussion über die Varietäten des Deutschen bereits wahrnehmbar ist.

\section{Exkurs zur Plurizentrik und Variation des Deutschen}

Jede natürliche Sprache ist gekennzeichnet durch einen gewissen Grad an Variation, die auf allen sprachlichen Ebenen und in allen Kommunikationssituationen erscheint, selbst die von der Schriftsprache getragene Form der Standardsprache ist nicht frei von Variation. Die sprachliche Variation im Deutschen ist wahrscheinlich der erste auffallende Eindruck für alle, die sich in den betreffenden deutschsprachigen Gebieten für längere oder kürzere Zeit aufhalten. Darüber hinaus ist auch mit dem charakteristischen plurizentrischen Charakter des Deutschen zu rechnen (vgl. Ammon 1995 und 2016), der sich auf die geographisch kompakten deutschsprachigen Gebiete in der Mitte Europas erstreckt - anders als im Englischen, Spanischen und Französischen, deren Plurizentrizität sich auch auf Gebiete außerhalb Europas, auch auf Übersee ausdehnt. Im Prozess des Erlernens von mono- und plurizentrischen Sprachen gibt es wohl keinen gravierenden Unterschied, doch ist damit - insbesondere im Ausland zu rechnen, das bei den plurizentrischen Sprachen eine Reihe von historischen, kulturellen, politischen und sprachgeschichtlichen Kenntnissen unentbehrlich sind, um die Sprache richtig gebrauchen zu können.

Die nationalen Standardvarietäten des Deutschen, das österreichische Deutsch, das Schweizerhochdeutsch sowie das Deutsche in der Bundesrepublik sind eigens kodifiziert, normiert, gleichrangig und haben einen auf die betreffenden Länder und Sprachgebiete bezogenen Geltungsbereich. Eine staatsorientierte Eigensprachlichkeit zeigen die Sprachgebrauchsweisen z.B. in der Schweiz und in Luxemburg, gleichzeitig sind sie auch Abbild der großräumigen Dialektlandschaften. Für Varietäten allgemein, so auch für die nationalen Standardvarietäten gilt m.E., dass sie kognitive Wissensbestände darstellen und

${ }^{6} \quad$ Das wird in den meisten Sprachprüfungen (in Ungarn) noch verlangt, erwartet. 
soziale Funktionen haben, auf diese Weise „leisten Varietäten einen Beitrag zur Konstruktion der Identität der Sprecher und deren Kategorisierung“ (Dovalil 2010: 45). Dass Lernende, Studierende diese Funktion von Varietäten erkennen können, bedeutet einen langen Weg, vor allem aber persönliche, hautnahe Spracherfahrungen auf den deutschen Sprachgebieten oder zumindest auf einem der Sprachgebiete.

Anhand des plurizentrischen Charakters der Sprache kann eine Sprachaufmerksamkeit und eine Sprachbewusstheit bei Lernenden und Lehrenden entwickelt werden, anders formuliert ist es wichtig, die Unterschiede der drei nationalen Standardvarietäten und ihre unterschiedlichen Verwendungsbedingungen auch im Studium den Lernenden/Studierenden im DaF-Bereich zu erläutern, damit sie diese Kenntnisse in ihrem Sprachgebrauch zumindest auf rezeptiver Ebene umsetzen können. Ohne auf einzelne Details der nationalen Standardvarietäten einzugehen, sei hier nur angedeutet, dass für Studierende, Lernende des Deutschen die Lexik im Österreichischen Deutsch (Austriazismen genannt) auch die suprasegmentale Ebene oder z.B. manche Differenzen im grammatischen Bereich (Artikel, Auxiliaria haben und sein) besonders auffallende Merkmale darstellen. Im Sprachgebrauch in der deutschsprachigen Schweiz sind es die Helvetismen sowie die konsequente diamediale Trennung (gesprochen: Dialekte, geschrieben Schweizerhochdeutsch) der Varietäten im Sprachgebrauch und nicht zuletzt im Sprachgebrauch der Bundesrepublik ist es das bekannte Nord-Süd-Gefälle, die regionalen Sprachgebrauchsstandards und die daraus resultierenden Differenzen auf der lexikalischen, grammatischen und pragmatischen Ebene. Es muss auch beachtet werden, dass es zwischen den drei nationalen Standardvarietäten insbesondere in Grenzgebieten z.B. im Südosten der Bundesrepublik und Österreich fließende sprachliche Übergänge gibt, also keine strikten sprachlichen Grenzen existieren. Dafür ist ein Beispiel das standardsprachliche Wort Junge, wofür als Alternative im Südosten Deutschlands in der Alltagssprache eher das nicht standardsprachliche Bub/ Bube verwendet wird, das im Österreichischen Deutsch als Standardwort gilt. 


\section{Bestandaufnahme des gegenwärtigen Normverständnisses}

(1) Nach einer ziemlich lange währenden Tradition von Gewöhnung an die präskriptiven Normen wurde eine Kompetenz im schriftsprachlichen Bereich erreicht, „die es auch ermöglicht, dass sich die Sprecher eine Domäne entsprechender Mündlichkeit dazu erobern“ (Eichinger 2011: 13). Der gesprochene Standard, der kodifiziert und relativ invariant ist, bedeutet für die Lehrenden eine sichere Basis, auf die sie in Zweifelsfällen oder bei Veränderungen zurückgreifen können. Doch die im gesprochenen Deutsch wahrnehmbaren lockereren Formen, die Destandardisierungstendenzen, zeigen, verweisen darauf, dass die Normen der Schriftlichkeit und Mündlichkeit nicht gleichgesetzt werden dürfen (vgl. Ziegler 2011: 71), denn in der geschriebenen Sprache herrschen Normen stärker als in der gesprochenen.

(2) Der Gebrauchsstandard, der von regional- und alltagssprachlicher Variation und Abweichungen vom Standard stark beeinflusst ist und der vor allem durch die Medien bekannt und verbreitet wurde, ist nicht einheitlich und nicht kodifiziert. Lehrende halten sich eher an kodifizierte Normen als an Abweichungen, Veränderungen und Variation, weil sie sich die Frage stellen: Woher nehme ich die Informationen darüber, wie flexibel die Sprachnormen sein können und wieviel Variation im Unterricht zugelassen werden darf? Die „Wechselbeziehung zwischen den Sprachnormen (Kodex-orientiertes Sprachverhalten) einer Standardvarietät und dem alltäglichen, aber auch öffentlich-offiziellen Sprachgebrauch einer Gemeinschaft in Wort und Schrift (usage-based Agieren), in dem sich neu herausbildende subsistente Regularitäten oder Normen widerspiegeln" (Felder 2003: 482), darf nicht ignoriert werden.

(3) Zuletzt können die Verschiebungen und Überschneidungen zwischen Gesprochenem und Geschriebenem Probleme im Unterricht bereiten, bzw. der Umstand, dass der Sprachgebrauch maßgebend von kommunikativ-situativ-funktionalen Parametern bedingt wird, die die jeweilige sprachliche Gestaltung und damit einhergehend auch die soziale Rolle des Individuums in der Gesellschaft bestimmen. 


\section{Fazit}

Die meisten Schwierigkeiten im Umgang mit Norm und Variation ergeben sich im Deutschunterricht in Ungarn m.E. aus folgenden Problemen:

- $\quad$ die Auffassung der Norm als etwas Starres, das Festhalten an präskriptiven Vorstellungen, da die Sprachrealität noch viele Unsicherheiten bedeutet,

- das Erkennen und die Handhabung der medialen Verschiebungen und Überlappungen von sprachlichen Äußerungen in der Unterrichtspraxis (man wird danach beurteilt, wie man sich äußert) und das Erkennen, dass nicht nur Sprache unterrichtet wird, sondern Sprache und Kultur zusammengehören,

- $\quad$ die didaktische Aufbereitung der sprachlichen Varianz auf allen (oder zumindest wesentlichsten) Ebenen des Sprachsystems und deren Implementierung in den benutzten Lehrmaterialien,

- $\quad$ der Leistungsdruck der Lehrenden und Lernenden im Ausland, ein möglichst einwandfreies Standarddeutsch zu vermitteln bzw. zu erlernen und im Abitur und den sonstigen Prüfungen gute Ergebnisse zu erreichen ${ }^{7}$.

Genauso wie sich die Sprachhandlungsmuster von Zeit zu Zeit ändern, genauso vollzieht sich eine Variabilität der Textsorten der Gegenwartssprache. Wann, auf welcher Schulstufe und auf welche Weise diesen Veränderungen Rechnung getragen wird, ist eine Frage der Lehrplangestaltung der einzelnen Schultypen im betreffenden Land und Frage der Gestaltung der lokalen Lehrpläne.

Statuierte Normen bilden schon jahrzehntelang das Kernstück des Fremdsprachenunterrichts und in der Deutschlehrerausbildung, demgegenüber stand die Vermittlung der Routinen, die für den Sprachgebrauch unentbehrlich sind, lange Jahrzehnte im Schatten der Unterrichtspraxis. An diesem Punkt scheint mir der Terminus technicus der transitorischen Norm von Feilke (2015) sehr angebracht zu sein, der andeutet, dass die im Unterricht vermittelten Normen

$\mathrm{Zu}$ der Lehrplangestaltung an zweisprachigen Minderheitenschulen in Ungarn vgl. Leitbild (2010), Kompetenzmodell (2010), Rahmenlehrplan (2010), der Nationale Grundlehrplan (2012, der neue ist im Entstehen). 
als Übergangsnormen betrachtet werden sollen, die sich erst im Laufe des Lernprozesses bei den Lernenden stabilisieren und sich zu einer richtigen Gebrauchsnorm entwickeln werden. Aus dieser Sicht betrachtet, wird die Lücke zwischen gesetzten Normen und Gebrauchsnormen etwas geringer ausfallen.

\section{Literatur}

Ammon, Ulrich (1995): Die deutsche Sprache in Deutschland, Österreich und in der Schweiz. Das Problem der nationalen Varietäten. Berlin / New York: de Gruyter.

Ammon, Ulrich et al. (2016): Variantenwörterbuch des Deutschen. Berlin / Boston: de Gruyter.

Berend, Nina / Knipf-Komlósi, Elisabeth (2006): Sprachliche Variation als Herausforderung für den Deutschunterricht in Osteuropa. In: Neuland, Eva (2006): Variation im heutigen Deutsch. Perspektiven für den Sprachunterricht. Frankfurt a.M.: Peter Lang, 161-175.

Busse, Dietrich (2006): Sprachnorm, Sprachvariation, Sprachwandel. Überlegungen zu einigen Problemen der sprachwissenschaftlichen Beschreibung des Deutschen im Verhältnis zu seinen Erscheinungsformen. In: Deutsche Sprache 34 (4), 314-333.

Davies, Winifred (2006): Normbewusstsein, Normkenntnis und Normtoleranz von Deutschlehrkräften. In: Neuland, Eva (Hrsg.): Variation im heutigen Deutsch. Perspektiven für den Sprachunterricht. Frankfurt a.M.: Peter Lang, 483-491.

Davies, Winifred / Langer, Nils (2013): Die Sprachnormfrage im Deutschunterricht: das Dilemma der Lehrenden. In: Plewnia, Albrecht / Witt, Andreas (Hrsg.): Sprachverfall? Dynamik-Wandel-Variation. Berlin: de Gruyter (IDS-Jahrbuch 2013), 299-321.

Dovalil, Vit (2006): Sprachnormwandel im geschriebenen Deutsch an der Schwelle zum 21. Jahrhundert. Frankfurt a. M.: Peter Lang.

Dovalil, Vit (2010): Zum Begriff, Varietät' und dessen Verflechtung mit ,Norm und ,Stil'. In: Gilles, Peter/Scharloth Joachim/Ziegler Evelyn (Hrsg.): Variato delectat (Empirische Evidenzen und theoretische Passungen sprachlicher Variation. Frankfurt a. M.: Peter Lang (Vario lingua 37), 45-47. 
Durrell, Martin (2006): Deutsche Standardsprache und Registervielfalt in DaF-Unterricht. In: Neuland, Eva (Hrsg.): Variation im heutigen Deutsch. Perspektiven für den Sprachunterricht. Frankfurt a.M.: Peter Lang, 111122.

Durrell, Martin (2012): Zur Relativierung von hochsprachlichen Normen in der deutschen Sprache der Gegenwart. Der Blick von außen. In: Günthner, Susanne et al. (Hrsg.): Kommunikation und Öffentlichkeit: Sprachwissenschaftliche Potenziale zwischen Empirie und Norm. Berlin / Boston: de Gruyter (Reihe Germanistische Linguistik 296), 89-105.

Eichinger, Ludwig M. (2009): Begrüßung: Vom grammatischen Wissen und seiner vernünftigen Verwendung. In: Konopka, Marek / Strecker, Bruno (Hrsg.): Deutsche Grammatik: Regeln, Normen, Sprachgebrauch. Berlin: de Gruyter, 1-8.

Eichinger, Ludwig M. (2011): Normprobleme, oder: Variation ist sinnvoll. Überlegungen zum heutigen Deutsch. Akademie der Wissenschaften und der Literatur Mainz. Stuttgart: Franz Steiner Verlag.

Feilke, Helmuth (2015): Transitorische Normen. Argumente zu einem didaktischen Normbegriff. In: Didaktik Deutsch 20 (2), 115-135.

Felder, Ekkehard (2003): Das Spannungsverhältnis zwischen Sprachnorm und Sprachvariation als Beitrag zu Sprach(differenz)bewusstheit. In: Wirkendes Wort 53 (3), 473-499.

Gloy, Klaus (1998): Zur Realität von Sprachnormen. In: Der Deutschunterricht 50 (3), 14-23.

Gloy, Klaus (2004): Norm. In: Ammon, Ulrich et al. (Hrsg.): Sociolinguistics. Soziolinguistik. Berlin / Boston: de Gruyter (HSK 3.1), 392-398.

Idegennyelvi kerettanterv [Rahmenlehrplan für die Fremdsprachen] (2012).

Kompetenzmodell = Wurzeln und Flügel. Kompetenzmodell für den Deutschunterricht der bilingualen deutschen Nationalitätenschulen in Ungarn (2010). Budapest: Landesselbstverwaltung der Ungarndeutschen.

Leitbild = Wurzeln und Flügel. Leitbild des ungarndeutschen Bildungswesens (2010). Budapest: Landesselbstverwaltung der Ungarndeutschen.

Miskei, Réka / Müller, Márta (2017): Welche grammatischen Nachschlagewerke benutzen Studierende? Ergebnisse einer Online-Befragung. In: Jahrbuch der ungarischen Germanistik 2016. Budapest / Bonn: GuG / DAAD, 119133. 
Müller, Márta (2010): Die Situation des Deutschunterrichts in Ungarn. In: Kosztrzewa, Frank / Rada, Roberta unter Mitarbeit von Elisabeth Knipf-Komlósi (Hrsg.): Deutsch als Fremd- und Minderheitensprache in Ungarn. Historische Entwicklung, aktuelle Tendenzen und Zukunftsperspektiven. Hohengehren: Schneider Verlag, 74-96.

Nemzetei Alaptanterv [dt. Nationaler Grundlehrplan] (2012). In: Magyar Közlöny [dt. Ungarisches Bulletin] 55. 4. Juni 2012.

Neuland, Eva (1998): „Sprachnormen“ - kein Thema mehr? In: Der Deutschunterricht 50 (3), 4-13.

Rahmenlehrplan = Wurzeln und Flügel. Rahmenlehrplan für den Deutschunterricht der bilingualen deutschen Nationalitätenschulen in Ungarn (2010). Budapest: Landesselbstverwaltung der Ungarndeutschen.

Stridova, Nela (2015): DaF-Lehrer als Normautorität am Beispiel eines tschechischen Gymnasiums. In: Sichova / Katerina Krapp / Reinhard Rössler / Paul / Dovalil, Vit (Hrsg.): Standardvarietät des Deutschen. Fallbeispiele aus der sozialen Praxis. Berlin: Logos Verlag, 117-133.

Ziegler, Evelyn (2011): Subsistente Normen und Sprachkompetenz: ihre Bedeutung für den Deutschunterricht. Bulletin suisse de linguistique appliquée 94 [Schweizerisches Bulletin für Angewandte Linguistik], 69-85. 